\title{
TUMORES MALIGNOS DE LA VULVA
}

(Estudio estadistico hecho en el Instituto Nacional de Radium)

\author{
Doctores REYNALDO ORJUELA GOMEZ y GUILLEMO LOPEZ ESCOBAR, \\ del Servicio de Ginecologia de la Universidad Nacional \\ y del Instituto Nacional de Radium
}

Por la gran variedad de tejidos, tante histologica como embriológicamente considerados que presenta la vilva. numerowo tipos de tumores nalighw pueden desarrollarse a sus exponsas. Eston diversos tipos deben ser teniule mus en cuenta ya que difieren entre si por su apariencia clínica, o bur si exclurión. sil pronóstico o su trataminte. I en muehas ocasiones por twass fllos simnltáneamente.

Entermedad it Bomen.

Ev un tipo especial de disqueratosis que oresenta en ocasiones en la regía rulvar. esperiamente en la época do la menopansia. Clínicamente se caraiteriza por la aparición de manchas que erolucionan lentamente acabando por uleerars e infiltrar los tejidos vecino y finahmente adquieren el aspec. to típir de los carcinomas. Histolónicamente se caracteriza por un espesamiento de las capas epiteliales, con hipertrofia de los mamelones interpapilares. deformación de lạs células altas de la capa basal y variedad de forma $y$ de colocación de las células de la capa espinosa. que presentan además dos o tres núcleos tumefactos o con disgregación de la eromatina. Por su eyo- lución histológica es llamado por Nowak (45) "earcinoma intraepitelial no invasivo" o carcinoma "in situ". Lunin (36), cita un caso de enfermedad de Bowen complicada con un eareinoma epidermoide grado III. Las estadístiass del Instituto de Radium. que nósctros recogimos. traen un caso (Historia numero 1.249), de edisqueratosis de Bowen de localización rulvar.

\section{Enfermedad de Paget.}

Es una enfermedad de evoluciôn may lenta, caracterizada clínicamente por la presencia de una costra seea muy adherente que posteriormente se nleera Y termina, al cabo de algunos años, transformándose en un típico carcinoma. Los estudios histológicos realizadós ultimamente, han confirmado al pare. cer la sospecha que se tenía de que esta entidad fuera primitiramente cancerosa. más propiamente una metástasis intraepidérmica de un cáncer subyacente (73). Se presenta en la mayoría de los casos en el pezón. habiendo en realidad. pocos casos extramamarios perfectamen te estudiados. Los casos mamarios pare cen ser metástasis de cánceres primiti-y vamente localizados en los conductos 
xiretores del pezón (44. 50, 56). asi como los extramamarios tienen su más probable origen en las glándulas suderípatras de la variedad apoerina $(55$. que se localizan principalmente en la asila y la región anogenital. Histolórmamente la caracteriza la presencia d. ...lnlas especiales, llamadas rélulas d. Paget o Psorospermas (21), gine se pr.. sentan aisladas o agrupadas en montones en el espesor de la epidermis: sou rodondeadas, excavadas en forma di. celdillas, con nucleo hipercromático ! eitoplasma claro. Respecto a su incidencia en la vulva Weiner (73), en su $\ldots$ tudio estadístico, cita un total de 16 casos de enfermedad de Paget extraniamaria perfectamente comprobada, de lecuales 9 o sea un $56.24 \%$ estaban localizarlos en la vulra. Aquí se han diagnosticado hasta el presente 12 casos de enfermedad de Paget (10 en el Instituto de. Radium, y dos. tratados con radiun. que fueron vistos por el Profesor Alfonso Esguerra Gómez). De ellos. 10 estaban localizados en el pezón $y$ don en las extremidades inferiores.

\section{Sircoma.}

Es un tumor que muy raramente si desarrolla en la vulva: Kehrer 32 encontró, revisando las comunicaciones al respecto, sólo 75 casos. $61 \%$ de los euales se habían formado en los grandes labios. Es o circunserito o difuse y no invade la piel en sus comienzos. pero produce metástasis muy precocts r sus recidivas locales son mur frecuentes, por lo cual se le considera de gran malignidad. Se han descrito rarios tipos histológicos de sarcoma: liposarcoma, fibrosarcoma, hemangiosarcoma, miosarcoma, mixosarcoma y linfosaŕcoma, siendo este último el más raro rá que sólo se han descrito dos casos
(27, 65). La edad más comín de inci. dencia, según Taussig. es la de 40 años. El pronóstico de estos tumores es muy malo, dada la precocidad de las me tástasis por vía sanguínea, y el trata. miento es quirúrgico en los casos pre. cocee. usándose los rayos $\mathrm{X}$ como palia. tivo n los casos avanzados. Keliper (32) no reporta curas de cinco años El sar ona vulvar puede provenir de fibr mas degenerados; 1 n caso en nue. ve, squín Folsome (24).

\section{Wetamina.}

Este tumor maligno procede como lo dijimos anteriormente, en un $83.91 \%$ de los casos de nevus pigmentados.

Es sin duda, el más maligno de los tumores que se pueden desarrollar en la regićn vulvar, debido esto a lo pre. ‘oz y rariado de sus metástasis, ya q!! práticamente puede invadir eualquier region del cuerpo: Kehrer (32) los ha110 dos easos con sobrevivencia de más de tres años en un total de 82 casus andlizados por él. Respecto a su incidencia en relación con el sitio de in. plàtación primitiva del tumor Pack $y$ Jivingston (47) dan la siguiente en orcis descendente de frecuencias: (1) Grindes iabios; (2) Pequeños labios: (3) Clítoris; (4) Vestíbulo; (5) Uretra. y (6) Monte de venus. Los de los per ueños labios se desarrolian casi siem. pre en sn tercio anterior que es la porción más pimentada. La iniçiación de la malignidad en un nevus-pigmentado se puede manifestar de tres maneras diferentes: (2) (I) Puede haber no carubio apreciable en el lunar primitiro o puede no descubrirse la lesión inicial. caracterizándose el comienzo clínico por un agrandamiento de los ganglios linfáticos regionales. (II) Comienza por un ensanchamiento del tumor 
primitivo que posteriormente puede ulcerarse y luégo cieatrizar pareialmente, sangrando intermitentemente. (III) la primera manifestación de malignidad observada es la aparición de numerosos tumores pigmentados en la niel que rodean el lunar primitivo. De estas tres formas de iniciación, la más maligna es lit ultima. en la cual el comienzo es sc. guido rápidamente por amplias metástasis y muerte por invasión viseral. Isos sintomas más comumes son el prurito y la hemorragia, debida esta última a lo vascularizados que son y a lo delicado de su estruetura. "El pronóstico, como ya lo dijimos, es muy malo, y el tratdmiento debe ser la vulvectomía precoz. La mayoria de los autores consideran que la linfadenectomía inguinal. en caso de melanoma, es inútil, pues debido a su alto grado de malignidad, cuando el tumor ya ha comenzado a invadir los linfáticos el tratamiento quirúrgico no podrá detenerlo. Es importante saber que el embarazo farorece mucho el desarrollo de estos neoplasmas por lo cua] aconseja Taussig (62) la extirpación, en el momento del parto, de cualquier nevus sospechoso que se localice en li región vulvar. Respecto a la frecuencia del melanoma en relación con los otros cáneeres de la rulva. las estadisticas de la mayoría de los autores le da $u$ porcentaje que varía más o menos entre el 1 y el $2.5 \% \quad(65,36,48)$, aunque Smith y Pollack encontraron una incidencia de $11.1 \%$ (25 en 228 casos vis. tos). Entre nosotros la incidencia ha sido de $3.64 \%$ (2 casos ent un total de 55). La edad más común encontradi por Taussig (62) ha sido 54 años; en el Instituto de Radium se han presentado dos casos de melanoma vulvar, que tenian 45 y 65 años.

\section{Trratomas.}

Son rarisimon I, wasm deseritos no llegan a la dorena. Taussig relaia cuatro. reportados por distintos autores, en su obra "I)isease of the Vulva" y cree que muchos de las tumores descritos "omo mixomas sorl probablemente de wigen teratomatus. Estos neoplasmas, ammque histoligiomente malignos se pucden considerar romo relativamente benimos. pues arean muy letitamente y es raro que produzan metástasis.

\section{Adrusoreinoma}

El adenocarcinoma se presenta en la vulva con alguna frecuencia, la mayoría de las reces desarrollado a expensas de las glándulas de Bartholin (17, 65), Y en ocasiones de las sudoríparas; es un cáncer bastante raro aunquẹ más frecuente que el melanoma. Clímieamente el desarrollado en una glándula de Bartholin s carateriza en sus comienzos por una masa subdérmica, dura, situada en el espesor del gran labio, en el lugar correspondiente a la glándulla, de tamaño variable $y$ evolución en : un comienzo lenta: posteriormente aleanza las capas superticiales de la piel produciéndose a su nivel una ulcerarión que sigue después una evolución semejante a la del epidérmico de forma ulcerosa.

El hidradenoma maligno es también subdérmico en sil comienzo, caracterizándose por la presencia de nódulos que ran creciendo paulatinamente hasta abrirse a la piel como el adenocarcinoma de la glándula de Bartholin. Este illtimo es un cáncer muy maligno, ya que hace rápidas metástasis intrapelvianas (5 de 6 casos de Taussig); el cáncer de las glándulas sudoriparas es menos maligno. Histológicamente pue- 
den presentarse dos tipos (14): el de mu varcinoma cilíndrico altanente diferenciado (adenoma maligno), con formación de cavidades glandulares con revestimiento monoestratificado, o bien el de un carcinoma cilíndrico con cavidides irregulares cuyo epitelio, a menitlo estratificado, tiende al relleno de lás glándulas y a la producción de cordones sólidos (adenueareinoma verdadero o carcinoma ciludrico semidiferen(ciado). Debe teners tn enenta que algunos de los c*arcinomas de la glómdula de Bartholin son de tipe escamorelular por originarse en su conducto excretor (12, 33, 57). La sintomatología en su comienzo se reduce a la presencia del tumor subdérmico, y en la etapa de ulceración tiene sintomas semejantes a los que presenta el carcinoma escamoceHular. de los cuales hablaremos al tratar día éste.

El pronóstico no es favorable. por sus tempranas metástasis. el tratamieutu. siempre que sea posible debe ser quirúrgico radical. Es raro que aquí no se haya presentado aún un solo caso de este tipo de cáncer. ra que es más frerturnto que el melanoma $(46.65)$. del cual sencontraron dos casos.

\section{rascinama epidermoide.}

Es el tmor maligne más frevllentrnente encontrado en la vulva. variando su porcentaje entre el 80 y el $9 i^{\prime} ;$ ite los casos (9. 50.65.66.67). Es relativamente el menos maligno de todos los tumores cancerosos que se desarrollan en los órganos genitales esternos feineninos, especialmente la rariedad vaso-celular. Como ya lo afirmantos antes, la leucoplasia $y$ la sífilis son las phtidades que más favorecen su aparición. Respecto a su localización primitiva ésta se hace en orden de frecuen- cia deseendente en: (1) Grandes latbios; (II) Pequeños labios; (III) Prepucio; (IV) Vestíbulo y región perihineneal; (V) Horquillat, $y$ (VI) Grande clitoridiano. (28, 45, 61, 62).

Clínicamente el carcinoma epidernooide se caracteriza por tres formas diferentes: una forma ulcerosa, endofitica. una forma vegetante. exofítica y una forma infiltrante difusa. La ulcerosa se caracteriza como lo indica su nombre. por la presencia de ma ulceración de bordes levantados, que se extiende tauto en superficie como en profumdidad; el fondo de la úlcera está formado por tejidos necrosados que despiden un olor fétido pronunciado y la base presenta una forma de coliflor, de consistencia blanda, color rojo amarillento $\mathrm{y}$ base relativamente poco infiltrada. La característica de la forma difusa es su gran infiltración, que se extiende a todos los tejidos vulvares obstruyendo el introito vaginal $y$ el meato uretral. y pudiendo llegar en profundidad hasta el hueso; la zona infiltrada puede ulcerárse secundariamente. De las tres formas ésta última es la más rara y la menos maligna es la exofítica (14, 63. 23), que es la más común en las riejas.

\section{Anatomía Patológica.}

Histológrcamente y siguiendo la clasificación americana, podemos diviciir los carcinomas epidermoides en espinncelulares y vaso-celulares, siendo mu.ho más frecuentes los primeros: 90 a $95 \%$ $(46,58,47)$.

El grupo éspinocelular puede dividirse, segúm el grado de diferenciación celular, en los mismos grados que los cánceres cutáneos de otras regiones dei cuerpo. De ellos a variedad más fre‘uente es el tipo II. altamente diferenciado, de células anchas y poliédricas de forma- 
ción colmmnar y gran tendeneid a producir grobos cómeos; el estroma siempre muestra geacción inflamatoria ni(.a muy extensa (46). El grupo vasocelular se caracteriza por su immadurez y la no formación de globos córneos. Novak (45), menciona un grupo inter-medio, "transicional", entre éste y" el espinocelular. En los tumores que se forman a expensas del clitoris hare. Taussig (62), énfasis sobre su forma muy semejante al sarcoma, con células de tipo immaduro, embrionayias. (on nicleos muy coloreados y marcada a dad mitósica; son muy malignos, pero afortunadamente bastante raros.

\section{Indict de Malignidad.}

Taussig (61, 62), siguiendo a Froders, hace una clasificación histolćcica de los cuatro tipos según el grado de malignidad; para ello se base en la cantidad de anaplasia encontrada. prestando especial cánceres epidermoides, dividiéndolos en atención a la hiperplasia y rápido desarrollo celular. varidcjón en tamaño, forma y calidad de colo. ración de las células y sus núrleos, asi como también al grado de infiltración. sus tendencias, el número y carácter de las mitosis y la reacción del tejido conjuntivo. La clasificación es la signielite:

\section{Tipo 1 .}

En el cual las células son bien diferenciadas, con grandes áreas de epitelio pavimentoso bien formado, grandes zonas de globos córneos y pocas células atípicas o mitosicas.

\section{Tipo II.}

Epitelio pavimentoso también bien desarrollado, con ocasionales globos córneos, pero con un marcado aumento ("n las células atípicas, profundamente whreadary mostrando mitosis más numeromas.

\section{Tipo III.}

Células ačnadas en nidos medularo ron solo asionales áreas de epitelio de tipo frimentoso $y$ con plenitud A. nitesis.

\section{Tim $\mathrm{I}^{\circ}$.}

En el chal la estructura del tumor wta perilida, con células marcadamen$t:$ polimorfas. células gigantes $y$ contadas mitosis.

Tanssig 61), hizo estudios histológiveos siguiendo este índice en 58 de sus (asts. para averiguar su valor pronóstieo. (\%) el siguiente resultado: tipo I, Sipte casos: tipo II, treinta casos; tipo III. diez $y$ se is casos, $y$ tipo JV, eines racos. El índice de malignidad fue nuas baj: en los puramente epidérmicos que (u) fos clitoridianos y de Bartholin, siend. los de más alta malignidad los desarmollates en wha base sifilítica en muje. res jórenes (restíbulo) y los del grande clitoridiano.

Como se pulede ver, Taussig, trata de basar el pros ćstico de los cánceres vulvares en su zrado de malignidad histológien: aumqune sus resultados son bastante buenos : es la clasificación histológiea que nos ha parecido mejor, murhos son los autores $(48,54,58)$, que juzgan su valor muy relativo como acontece con la clasificación según el grado de malignidad clínica del mismo Taussig.

\section{Propagación.}

Hay tres maneras por las cuales puede propagarse un cáncer vulvar: (I) Por contimuidad (a) hacia la piel y 
moucas proximas; (b) hacia los órocanos recinos; (c) hacia el tejido eonjuntivo de la fosa isquiorectal o de los tabiques recto y uretro-vaginales y aún al paracolpio (tejido conjuntivo paravaginal! y (d) hacia los huesos de ia pelvis III Por vía linfática, siguien(li) en w raso de epitelioma ppidermojdit det lab labios y el prepucio el siguient* (amino: primera etapa. ganglios inguinales superficiales. Segunda etapa. ganglios inguinales profundos y terce. rif etapa ganglios ilíacos externos, hipafástricos y obturadores. Los cánceres, del clitoris (cuerpo y glande), se propagan por la vía linfática en: primera etapa, ganglios presinfisiarios $y$ retropúbicos, y segunda etapa, ganglios iliopélvicos a inguinales profundos. Los 4tilibulo ran por el siguiente camimo: a ganglios inguinales superficialos. de; donde siguen un trayecto semejante a los del gran labio, $y$ (b) ganqlios laterovesicales, hipogástricos e iliacos externos. Los de la horquilla vulvar si dirigen en su mayoría en una primo. „ra etapa a los inguinales superficiales 4.para seguir luégo un trayecto semejante a los linfáticos del resto de la superfirie cutánea de la vulva, pero algunos wectores van a los ganglios anorrecta19. La propagación linfática es, sin du- df algma. la más importante de tokas. n.illi Propagación a distancia. Se pue: fin presentar metástasis a la columma rertebral, bazo, hígado, etc. $(14,18$, 4.5).

\section{reasificacion clínica.}

Numerosas han sido las clasifica.jones clínicas que se han hecho sobre e' *arcinoma vulvar y podría decirse que cada autor sigue una propia, diferente a las demás. Mencionaremos en seguida las más importantes, comentando lué- go brevemente las ventajas y descentajas que a nuestro juicio pledan tener. e indicando la que nos parezua más “ompleta de todas ellas

\section{Clasificarion de Ber:en.}

(10) Este antor divido el cáncer vul. var en grupos: $19.20 .3054 \%$ en relación con la lesión primitiva. y en etapas: I. II y III, de arelterdo con la invasion de los ganglion regionales. De estas do diferente clasifirariones has. una sola, ron 12 diferentes tipos clíniros de cáneer. como signe: grupo 1?. tumores limitados a nna pequeña palte de la rulva un eentimetro de diámetrol. sin uleeración superficial o fijación profunda. firupo II tumores limitados a una parte de la vulva. con alceración $r$ infeccion secundaria y con $n$ sin ganglios inguinales infartados. Grupo III. tumor lowal yue se extienTe por fuera de la zona vulvar primitivamente afectada $y$ aun por fuera $d e$ la vulva nisma. Grupo $T$. tumores que se extienden más allá de la volva. pudiendo invadir hacia arriba la vagind $y$ smperficialmente el periné y la piel do las regiones rexinas. Etapa 1, ganglion pequeños móviles. bien cirrunseritos aunque blan os y obviamente inflamados. Erapa IT, qanglios anmentados to tamañ. inturados y con apariencia metastásica. Etapa III, ganglios hipertrofiados. Ia fijados e indudablemente invadidon.

\section{Clasificación de Carranza.}

Agrupa ol cáncer d la vulva. según el grado prolutive. on rnatro tipos diferentes: tipe I. lesiones localizitctar o superficiales, con repercusión gangirinar nula o apenas perieptible. Tipo II, lesiones de mayor extension o que infiltran más profundamente los teji- 
dos, con arenopatias remionales expe. riorizadas (ganglion duros. independien. tes y móviles). Tipo III. comprentie los rasos en los cuales la lesion loxal tiene importancia por su extensión o su invasión a los planos profundos, (con adenopatias inguinoerurales fijas. Tipo $\mathrm{IV}$, lesiones locales inextirpables por formar $\mathrm{m}$ bloune de toda la reción perineal, o no tan extensas pero con arlenopatías inguinormmales y pelvianas. la mayoría de las verens vegetantes a siluradas. a lo cnal se agrega nin périmo estado general.

\section{Clasificación de Palmer-sadugur.} Rrimhard.

Ellos clasifican este cáncer en tres crupos: grupo $1^{0}$ casos no tratados anteriormente en los cuales el tumor está limitado a la vulva. sin tener en euenta la extensión de éste. Grmpo go casos no tratados anteriormente. con evidencia clínica de invasión ganglionar. sea superficial o profunda. Grupo 3\%. rasos anteriormente tratados por virugia. irradiación o una rombinación de las los, haya o no metástasis ganglionares.

\section{Clasificación de Smith y Pollack.}

Es la siguiente: clase I, tumores y no muestran extensión: (a) por fuera del sitio primitivo al examen eliviro. (b) En los cuales los ganglios reoionales se disecan sin encontrarse invalidos al examen microscópico. Clase II. t:tmores con ganglios inguinales invadilos pero operables. como lo determina: (a) el examen clínico, y (b), Ja disec. ción de los ganglios con resultatos de biopsia positivos para cáncer.

Clase III, tumores inoperables, con ganglios inguinales fijos $y$ edina de los miembros inferiores.
Clasificación del subcomité de la ramepaña Anticancerosa del Imperio Britimico.

Tos divide en tres estados: Estado I. tlmor lmmitare a su sitio d, orizen, sin alenopatía concomitante. Dstad. II. tumor que ha invadido los tejidos ve. rinos, pero no en profundidad, pues no hay fijarión, con adenopatias novites. Estado III, tumor adherido a los pla. nos subyacentes, con amplia initira. •jón de los tejidos circunvecinos y adonopatía fija.

\section{Clasificación de Taussig.}

Divide los cánceres de la vulva en ein-o estados, de acuerdo con su evolucicial. Estado 1, carcinoma de menos de tres centímetros de diámetro, sin infiltración profunda, ni adenopatías. Estaôn "I: carcinoma de tres a siete centímetros le diámetro, sin infiltración profunda si adenopatía. Estado III: (a) carcinoma de más de siete centímetros de diámetro. sin infiltración profunda ni adenon::tías. (b) Carcinoma de más de sietr eentímetros de diámetro, con infiltración profunda o adenopatías móviles. Esiado TV, carcinoma propagado a la vagina. la uretra, el recto o el espacio sul.: público, o cualquiera de los estados anteriores con infiltración del tejido lular o ganglios inguinales adherentes. Estado $\mathrm{T}$, invasión de toda la vulva con infiltración vaginal o con granás metástasis inguinales ulceradas y netástasis a distancia. De los cinco Fstados considera como operables los tifs primeros.

De todas estas clasificaciones clínica: la que nos parece mejor es la do Taus. sig. que es bastante completa, ya que tiene en cuenta la mayoría de los diver. sos factores importantes que intervie. 
nen en la evolución clínica de estos neo. platanats, como son: la extensión de la lecion. el grado de infiltración y de inrasion $y$ el grado evolutivo de la metátasis ganglionares. Estos factores tienen pecial importancia en el criterjo ternifutice a elegir y sobre tocto en el protintico de la lesión en relación unin la posibilidades de éxito. Los re. sultafor obtenidos por él en un total de 47 asos clasificados de esta manera of los vuales se intervino, fueron los siguientes: 10 casos del Estado I. ope. rados ton una sobrevivencia de 10 " ser 100\%. 20 casos del Estado II, ope. radis con nueve sobrevivencias o $45 \%$, I0. ‘asos del Estado III, con cinco sobreviven ias o $\mathbf{5 0 \% , y}$ un caso del Estado TT qua no sobrevivió (61) Taussig, sigiverto sn clasificación clínica, halló en sutsestadísticas un $75 \%$ de casom operables. $y$ de los operados. 68 en tofal. fncontró un porcentaje de sobreviventa de $65 \%$, que es el más alto hasta ahora obtenido (65).

La clasificación de Berven nos parexp nur extensa y complicada, pues atund e tiene en cuenta más o menos los misn a factores que la de Taussig. hace (rin' thos nua agrupación menos simple. más difícil de interpretar. La de Carraicia. exolutiva también. es buend. aunitio stus diversos grupos no estái "tan" paramente delimitados como en la de Taussigr. La clasificación de Palmer - lánglesa nos parecen demasiado esquenáticas $y$ vagas, especialmente la nilinia. de manera que tanto el criterici pronóstico como el terapéutico se hace con ellas tal vez demasiado amplio. Eald Smith y Pollack, no es puramente clinica ya que en ella influye el diagnóstico anatomopatológico.

Debemos anotarle a todas las clasififationes mencionadas el no tener en cuenta un factor pronóstico importante: loralización primitiva del tumor.

\section{Simtomatologia.}

El eáneer vulvar es bastante pobre en sintomas y esperialmente en su etapa inicial; éstos son tan poeo llamativos a veces, que la paciente los pasa desaperr.ibidos o les presta poca atención. Es ya en los casos avanzados, cuando se presenta toda la grama de sintomas que nos van a indicar la propagarión cancerosa.

\section{Prurito.}

Fs mo de los más comunes sintomas del ráneer vulvar y como factor promostico tal vez el más importante de todos, pues se presenty te ordinario mucho tiempo antes (meses y hasta años), do comenzarse a desarrollar el tumor maligno. de manera que si so estudiara detenidamente el origen del pruritu rulvar en sus comienzos, especialmente en mujeres cercanas a la menopausia. podrian eritarse gran cantidad de futuros neoplasmas mulvares. Fue el sintoma inicial más comín $(51.09 \%$ de los casos) en las series de Palmer (48). sienclo el segundo en frecuencia, después de la percepeión misma de la lesión. en las estadístieas de Lunin (36) y Sinith y Pollack (5s). Sus características fueron ra deseritas al hablar de la leucoplasia.

Pero debemos recordar que sus caracteres principales son: la tenacidad de su persistencia. sus exacerbaciones nocturnas $y$ el poco efecto calmante del tratamiento médico sintomático. Los lugares en que con mayor frecuencia se presentan son el prepucio, el clítoris, los pequeños labios y la región anoperineal. Las estadísticas del Instituto de Radium. mencionan este síntoma solamente en un $10.53 \%$ de los casos, ocu- 
pando el tercer lugar en frecuencia despué de la peroperion de la lesión (ler. ligar) y la hemorragia (20 lugar). lo cual no está de acuerdo con otras estadísticas.

\section{Hemorragia.}

E- un sintoma que indiva un essio avanzado, aunque en los vasos intermedios pueden presentare teseargas stropurulentas tenidas de sangre. Se presenta como la hemorragia del enelio wterino. con oeasión de prytuños tramma. tismos contarte sexual, duehas, rased do). siendo de cantidad pequeña casi siempre; las hemorragian fuertes son raras (6:3). Las forma exofistitas produen hemorragia con mayor frecuencia que la endofiticas. Las estadisticas presentadas en este trabajo señalan a la hemorragia como el segundo síntoinit en frecuencia, aunque debe anotarse que las dos tercera- partes de estos casos se presentaron en parientes intratables. rasi siempre con invasión a la vagina. En rambio. las estadístias do los diver. sos autores indiean que ex un sintoma relativamente poen romín $136,48$. 50. 63 ).

Plujo.

E] flujo que provenga diretamente de la región afertada es relativamente infrecuente e indica nn estado de inflamarión e infectón sereundaria de la lesión. Es casi siempre seropurulento. a veces pintado de sangre. continuo y más o menos abundante. Debe tenerse eli cuenta que el flujo vaginal al irritar los tejidos vulvares puede dar origen a un rebelde prurito que se acen. tuará más aún con el rascado. Los diversos autores lo consideran raro y de los 62 casos del Instituto de Radium. sólo se encontraron cuatro con este síntoma, como antecedente inir.ial.

Dolor.

Síntona muy tardío, pues se presenta solamente cuando hay grandes metástasis ganglionares o invasión del periostio, tomando entonces las cararterísticas de los dolores cancerosos contiunos, fuertes, con exacerbaciones y nuly difíciles de tratar, pues a reces, aun con altas dosis de opiáceos, no logran ser calmados. Es por no ser dolerósas las lesiones malignas incipientes por lo que las pacientes les toman poco inte. rés, dejando transcurrir esparios de tiempo que son preciosos para el pronóstico favorable de su lesión sin harerse tratamiento alguno $y$ presentándose donde el médico cuando el caso está ya prácticamente perdido.

\section{Polaquiuria y Disuria.}

Son síntomas qué indican una invasión de la uretra y no son por lo tanto frecuentes en la etapa inicial del carcinoma rulvar, lo contrario de lo que pasa en el cáncer uretral en el cial son síntomas precoces. La dificultad para la miceión puede ir aumentando paulatinamente a medida que evoluciona el neoplasma, pudiendo llegar a jure. sentarse ma completa obstrucción de la uretra. Isa sensación de ardor a la nicrión es un síntoma que se presenta en ocasiones desde el comienzo dé la ajección, al pasar la orina sobre las zonas enfermas, irritándolas y macerándolas.

Perturbaciones rectales.

Se presentan algunas veces en forma de tenesmo $y$ pujo cuando el tumor invade las paredes del recto, pudiendo aparecer también la incontinencia de 
interias fecales en caso de ser destruíio el esfinter anal o también si so forma una fístula recto vaginal ; esto aparece, claro está. en casos ya avanzadísimos, especialmente en cánceres yriginados en la horqu illa o el vestíbulo.

Bisparennia - Edema A. los miembros inferiores.

Trussig $(61,63)$ cita algumos casos en los cuales el sintoma inirial fue la dispereunia, casos que probablemente se iniciaron en el vestíbul. En cuanto al flema de los miembros inferiores, se - presenta como consecuencia del bloqueo linfático originado por las metústasis a los ganglios inguinales $y$ es por lo tanto de muy mal pronóstico.

\section{Prescincia de la lesión.}

La gran mayoría de las veces, 70.1 ; . según Palmer (48), lo primero que llama la atención de la enferma es la presencia misma del tumor maligno $y$ ceneralmente, cuando esto sucede, el neoplasma lleva ya algún tiempo de evoJución y aun a veces ya ha hecho metástasis ganglionares. Lis estadísticas de las euales hablaremos posteriormente conenerdan a este resp cto con las dadas por otros autores. $(13,19,36,48,58$. 63 . $y$ dan un porcentaje de $54.39 \%$ de casos en que el síntoma principal inicial fur la aparición de la lesión canrerosa misma.

\section{Diagnóstico.}

Aunque el diagnóstico de certeza sólo lo da el estudio microscópico de la biopsia, clínicamente puecle hacerse o sos]echarse el diagnóstico de un neoplasma vulvar, de acnerdo con algunas caracteristicas que presentan los tumores rialignos desarrollados en esta región.
Estos caracteres son principalmente dos: en las formas exofiticas la sum friabilidad de los tejidos. pudiendo extraerse trozos del tumor con gran facilidad; y en las formas ulcerosas la marcada infiltración de los tejidos circundantes. Respecto a la hemorragia, aunque se presenta con alguna frecuencia no lo es tánto como en los cánceres localizados en otras regiones: con todo, en las formas uleerosas, que son las que relativamente sangran menos, el manipuleo produce con mucha frecuencia. pequeñas salidas de líquido hemáticos. lo, que no sucede con las úlceras de origen sifilítico o tuberculoso. por ejemplo (61). Puede también llegarse a un diagnóstico clínico siguiendo el eriterio terapéutico de eliminación, de acuerdo con los resultados obtenidos por los tratamientos que usualmente se emplean para curar las diversas lesiones entíneas de esta región.

Uno debe de tener siempre en miente el diagnóstico de cáncer vulvar. especialmente en las lesiones que no han cedido a otros tratamientos, I a pesar de los resultados negativos de las biopsias. Entre las historias que meneinnaremos más adelante hạ una (número 19.679). que confirma este acerto: en efecto, a esta paciente se le tomaron dos biopsias sucesivas que dieron resultados negativo para cáncer, pero con todo se signió sospechando la presencia de un neoplasma maligno; se hizo un tratamiento antisifilitico por tener Kann (x) y Mazzini ( $x x$ ), pero no se obruvieron resultados regresivos en la lesión; se insistió entonces en hacer una nueva biopsia cuyo resultado fue positivo para carcinoma espinocelular. grado II ; fueron pues, necesarias tres biopsias $y$ un tratamiento de prueba para llegar a un diagnóstico positivo. 
Respecto al diagnestico difereneial. debe hacerse con lesiones gire se asmm jen a las formas exofiticas o a las uhcerantes, pues la forma infiltrante difusa es muy característica.

Las formas vegetantes deben diferenciarse especialmente de las verrugav patpilomatosas que no sangran o lo hamen con difieultad $y$ son de consistencia firme. También deben tenerse en citilta las verrugas sifilíticas. semejanta las anteriores, pero de color vara.terístico, que ceden rápidamente al tratamiento antiluético: y las rulriti hipertróficas o elefantiasis vulvares. protducidas principalmente por el desasen r la filariasis y que aunque vegetantes. no presentan la facilidad para sangrar ni la consistencia característica de la lesiones malignas del mismo tipo 163 . En general estas formas exofiticas cancerosas presentan una mayor facilidad para el diagnóstico clínico que las formas ulcerosas endofíticas. $\mathrm{El}$ diagnixtico diferencial de estas últimas debe hacerse con todas aquellas afecciones que pueden presentar lesiones semejantes, pero especialmente con las úleera. sifilíticas $y$ tuberculosas. el linfograntloma venéreo, y a veces con el grannloma inguinal, el chancro blando $¥$ lat leishmaniasis. Las úlceras sifilíticas terciarias producen considerable infiltración pero, como ya lo dijimos. sangran con mucha más dificultad que los carcinomas, además de que sus bordes son acartonados, violáceos $y$ se encuriltran rodeadas de tejido cicatricial. sitio de antiguas úlceras (63); además las reacciones serológicas son fuertemente positivas. Las gomas sifilíticas tienden a ulcerarse evolucionando luégo como las úlceras ya nombradas. La úleera tuberculosa es muy rara, localizándose usualmente en, los labios y la región res- tibular: es de aspecto irregular, con bork cortados a pieo y fondo caseoso, y sin infiltración circundante marcada; su diagnóstico de certeza se hace no sólo por el examen microscópico, sino también por la comprobación del bacilo en los tejidos y por la inoculación al curí (45).

Entre los casos estudiados al hacer el presente trabajo, hay uno; el de la enferma I. C., del Hospital de San Juan de Dios, en el cual se constató microscópicamente la presencia simultánea de $u 1$ carcinoma espinocelular grado III $y$ una tuberculosis vulvar. La en. fermedad de Nicolás y Favre presenta viceraciones en la región vulvar muy rebeldes al tratamiento, de evolución crónica $y$ caracteres bastante semejantes a los de la forma ulcerosa del farcinoma de vulva, siendo muchas ve. res muy difícil hacer un diagnósticc diferencial entre las dos, fuera de que con frecuencia se ve coincidir estas dos afecciones (36); la reacción de Frei nos indicará la presencia de. un linfogranuloma venéreo. El proceso ulcerativo del granuloma inguinal es sirn' pre bastante extenso pero superficial, lo que lo diferencia de la ńlcera cancerosa que se extiende no sólo en superficie sino también en profundidad (63). Respecto al chancro blando, sus características son: su multiplicidad, pues casi nunca hay una sola ulceración, sing. varias diseminadas o confluentes, su erolución aguda, corta, con tejidos cireundantes inflamados pero no infiltrados, y lo dolorosas que son las lesiones; puede por lo tanto, diferenciarse más bien fácilmente de un proceso canceroso. La leishmaniasis americana puede ocasionar ulceraciones de evolución larga pero son de base poco infiltrada y pre. sentan un fondo granuloso caracterís- 
tien. cediendo a su tratamiento con fahidad (21).

\section{Pronóstico.}

El pronóstico del carcinoma vulvar, como el de casi todos los cánceres, deyende ante todo de la precocidad del diagnóstico; éste presenta en estos casos ba gran dificultad del falso "pudor de muthas mujeres, que sólo se deciden a consnltar al médico cuando ya es materialmente imposible hacer un tratamianto eficiente; a eso más que ninguwa otra causa se debe el que casi la mitat de los casos de cáncer vulvar, vistos en el Instituto de Radium (28 en un total de 62), hayan sido consideralos como intratables $y$, es a este punto al que debe dirigirse la propaganda edncativa anticancerosa, tratando de ineulcar en la totalidad de la población femenina, los grandes beneficios que para ella puede tener el examen médico periódico, divulgando especialmente todos aquellos síntomas premonitores y enfermedades predisponentes, como se ha becho en otros países, con resultados bastante apreciables. A dicha campaña deben adherirse no solamente las entidades oficiales $y$ particulares, sino también y especialmente los médicos, quienes deben aprender a distinguir précozmente toda la gama sintomática das afecciones malignas, pues muchas reces son ellos mismos los que, al instituir un tratamiento errado, hacen jerder a la paciente un tiempo precioso, Hevándola en muchas ocasiones, injustifri-adamente, a la misma muerte. Teniendo esto en cuenta la mortalidad de earcinoma vulvar podría verse des. uender considerablemente, ya que por lo menos in tres cuartas partes de los casos $(61,62)$, se eneuentra una base patológica la cual, de ser tratada ade- cuadamente por procedimientos miedicos o quirúrgicos. puede desaparerer antes de que, convertida en lesión cancerosa, se haga su pronóstico más reservado.

Respecto a los diversos factores que pueden modificar el pronóstico lel ráncer vulvar, podemos mencional los siguientes:

(I) Localización. Al parecer. cono tia sido comprobado por diversos autores (36. 58. 54. 65), el eareinoma que se desarrolla inicialmente en el clítoris. es; el de peor pronóstico: a éste le sipuen en orden descendente los cánceres de] vostíbulo y la glándula de Bartholin. siendo los menos malignos en este sentido los formados en los labios mulvares.

(II) Estado evolutivo. Claro está, y ya lo dijimos, el pronóstico de las lesiones iniciales es mucho mejor que el de las aranzadas; afectan especialmento dicho pronóstico las metástasis inguinales $y$ a distancia $y$ las propagaciones a la vagina la uretra y el recto $(6: 2.63)$. Siguiendo la elasificación clíni a de Taussig, podemos considerar com casos sin esperanza aquéllos que preventan metástasis a distancia o gangl mares ulceradas, o invasión extensa te los órganos vecinos.

(III) Tipo histológico. El tum or niàs maligno es sin duda el melanon a 63. 4i. 73 ), como en cualquier otri parte de la piel y son contadísimos los casos que muestran sobretivencia de más de einco años. A éste le siguen en malig. nidad el sareoma $y$ el adenocarimna, siendo. los menos malignos los carvinomas epidermoides, en especial el vaso celular. Respecto al grado de malignidad histológica de los cánceres pavimentosos está aún en discusión la importancia que pneda tener eomo valor pronóstico, pues mientras unos con 
Taussig a la rabeza, están en su fanor, otros (27, 40, 5o, no te conceden uingín valor.

(IV) Forma clínica. Se ha compro. bado que las formas exofíticats, en (•oliflor, son menos malignas que las infiltrantes y ulerosas. Fa yue e.ren con más lentitud y sélo se propawan a las zonas recinas en sus últimos e-tados.

(V) Tratamiento. Sin lugar a dudas. Yen eso están de atuerdo casi todos los autores. el pronistico de los casos tratados por eirugia radical (tip) BassetTanssig. es mucho mejor que el de aquéllos en los cuales se emplea el Radium, los Raỹos $\mathrm{X}$ o las simples escisiones, y asi por ejemplo Taussig (63, 6.5), tuwo un porcentaje de sobrevivencias de cinco años de $65 \%$ en los casos tratados por cirngía radical, según su técnica. y sólo 4.8\% en los tratados por Rayos $X$ o Radium : s Mekelrer. en 135 casos encontró un $15.6^{\circ}$; de maciones de cinen años en los tratados por Rayos $X$. Radium puneión o mulvectomía simple y un 59\% en los tratados ron vulvetomía radical y linfadene-tonía inguinal bilateral. La diferenıia es pues bien apreciable.

\section{Tratamiento.}

Podemos dividirle en eurativo y paliativo.

Tratamiento eurativo. Tres son los principales procedimientos empleados para tratar los eareinomas rulvares: Radium punción. Rayos X y cirugía. En un principio el tratamiento por irradiación (Rayos $\mathrm{X}$ y Radimn) fue el de elección._empleándose la cirngía sólo en raras ocasiones, pero poco a poco los estudios estadísticos fueron comprobando que era éste en realidad el tratamientu a elegir. Con todo aín quedan algunos partidarios de la irradiación
(13, 29), pero cada vez son menes. A este respecto debemos anotar que entre el público en general. y aun al. gunos médicos. hay un eierto temor por la intervención quirúrgica, pues ereeit que dado lo extenso de las reseceiones y la consecuente mutilación, quedan defectos que van a influir, esperialmente en pacientes jóvenes, sobre las relaciones sexuales $y$ el mismo embarazo. Esto es completamente falso, ya que las estadísticas de numerosos autores 16.5. $58,36,55)$, demuestran que tanto las primeras como el segundo, no son afertados en modo algumo por el tratamiento quirírgico. Entre los casos de que hablaremos más adelante hay dos que sustentan esta afirmación: el primero (historia número 19.679), paciente de 27 años, quien despnés de haberle sido practicada la operación tipo BassetTaussig, manifestó no encontrar ninguna diferencia en las relaciones sexuales antes $y$ después de ésta; y el segundo (historia número 19.179), que fue operada estando embarazada, sin haherse perjudicado por la intervención. su estado de gravidez, y quien está nuevamente embarazada.

\section{Irradiación.}

Incluímos dentro de este término los tratamientos hechos con Radium punción y los hechos con Rayos $X$, ya que sus resultados son más o menos seme. jantes.

En un principio se ereyó que dados los espléndidos resultados obtenidos en el tratamiento del cáncer del cuello uterino por medio de la irradiación, el cáncer vulvar reaccionaría de igual nianera, pero se ha comprobado que está muy lejos de ser así. La razón para ellos, según Taussig $(61,65)$, es doble: (I) El cáncer vulvar produce prococes 
matastasis ganglionares que rarmmente fueden ser destruídas por comploto. isando el Radium o los Rayos X. I tsit piel de la vulva es nomalmente nat! smsib'le a dosis relativamente pecint.. naas de Radimu o Rayos X, de manera the la rompleta destrucción de ma ril. era maliona es casi imposibles de realizar sin una consecuente necrosis de ho frjidos circundantes nuy dolorosa y extrusa. Con todo, el tratamiento por ir ta. liación puede eurar perfectamente un fáncer vulvar, de ello no hay dufla pro Ii) difíc.il es saber cuándo no en pusible liarer lo mismo, con mejor resiltade. empleando la cirugía. En muchas orasiones las radiodermitis. radioepidernitis $\mathbf{v}$ am necrosis ocasionalds por la irradiación producen tal malestar que se consigue in efecto rompletamente contrario al buscado, debiendo entones recurrirse a la cirugía para extirpar exas lesiones, con lo cual se obtiene una rápida desaparición de los lacinantem dolores, a pesar de la extensión de lat

Respecto a la acción que pueda trier heridas operatorias (62.63).

este tipo de tratamiento sobre las netástasis ganglionares. los resultados han sido muy diversos. pues en mins ouasiones se ha obtenido una dexaparieión completa de las infartaciones. en otras no sólo se han aumentado. sino que se ha producido su ulceración secundaria. Sobre la conveniencia del empleo de la jrradiación ganglionar inguinal como profiláctica, antes o después de la intervención quirúrgica, las opiniones están igualmente divididas. I Taussic. rpina que mientras no se demnestre su valor positivo como profiláctica es mejor no emplearla; esto debido a dos hechos principales $(63,64)$ : (I) el posihle aumento de la neerosis que casi siempre se produce en las heridas in- minales. debido a su gran extensión. (1) La auemia post-irradiación que vendría a sumarse a la producida por la interrención quirúrgica en si, repereutiendo directamente sobre el pronóstico. De aquí podemos concluír que soiamente en aqueilos casos en que está contraindicado el tratamiento guirúrgico, podrán emplearse la radiumpunción a los Rayos $\mathrm{X}$, como terapéutica enrativa. lo contrario de lo que sucede er casos de cáncer uretral o perimeal, en los cuales es el tratamiento de eleccion. como lo veremos más adelante. Respercto a las dosis empleadas como eurativas, son muy variables, pues dependen del estado de la lesión en sí (evolución, extensión, metástasis, ete.) firaves (27), empleó en sus casos una tosis de radium que osciló entre 1.51 m. c. d. y 36.240 m. c. d., siendo la dosis nsual 11.332 a $12.080 \mathrm{~m}$. c. d. y ma dosis total de Rayos $\mathrm{X}$, que varió entre 800 y 3.200 r', siendo la más empleada $1.600 \mathrm{r}^{\circ}$.

\section{rirugía.}

Debido a la relativa poca frecuencia del carcinoma vulvar (4\% de todos los cánceres genitales femeninos, segín Taussig), no es de extrañar que los procedimientos quirúrgicos para tratar1o sean numerosos, variando especialmente en la extensión de la resección. En efecto, mientras unos hacen mina simple extirpación de la lesióe o rulrectomía parcial, otros hacen una vulrectomía radical extirpando además los ganglios inguinales superficiales, uni o bilateralmente $(48,54,72)$; un tercer grupo hace vulvectomía radical con linfadenectomía inguinal bilateral, resecando tanto los ganglios superficiales como los profundos $(7,36,22,15$, 41. 58. 61); $\mathrm{m}$ cuarto grupo no se 
contenta mon esto solamente, simo que race además la extirpación de los ganglios ilíacos internos y primitivos $13 \%$, (0). I hay finalmente un grupo, encabezado por Robertson (51), que va aún más lejos haciendo además la extirpación de las cadenas ganglionares. lumbares al mismo tiempo que una simpaticectomía lumbar, uni o bilateral, semín el caso. De todos estos procedimientos el más conveniente, a nuestro modo de ver, es el preconizado desdo hace bastante tiempo por Basset, modificado posteriormente por Taussig, quien ha sido tal vez la primera autoridad en cáncer vulvar; claro está que esta térnica, que se comenzó a emplear riesdle hace tres años en el Instituto de Radium, no se puede usar sistemáticamente en todos los casos, pero sí en la maroría. teniendo la ventaja de que es el procedimiento que une a una resección lo más conservadora posible un porcentaje de supervivencia superior a los obtenidos con los demás procedimientos (38, 41, 58, 63). En realidad las técnicas en que no se hace linfadenectomía parecen estar abandonadas casi por completo, debido a los bajos resultados obtenidos con ellas $y$ en cuanto a las operaciones más radicales que las de Basset-Taussig, las estadisticas han demostrado que no tienen sobre ella ninguma ventaja $y$ sí en carrbio un porcentaje de muerte post-operatoria mucho más alto (15 a $20 \%$, según Kehrer).

Operación de Basset, modificada por Taussig.

Consiste esencialmente en una vulvectomía radical con linfadenectomía inguinal bilateral, superficial $\mathbf{y}$ profunda. En un principio se hacían las dos intervenciones a través de una sola in- cisión que resultaba por ello muy ext'usa, difienltándose extraordinariamento su cicatrización. Debido a esto, y teniendo en enenta la extrema rareza di. las metástasis a la piel de la región lateral del pubis, Taussig (61, 62, 63 resolvió dejar un puente eutáneo a calla lado entre la herida vulvar y la ingu nal, haciendo en vez de una gran in.-i. sión tres incisiones más pequeñas. aum en los casos en que efectuaba simultineamente las tres interventiones, con magníficos resultados. Como regla s t... niendo en euenta lo prolongado de li intervención $y$ el estado físico cani siempre deficiente de las pacientes, se bace la intervención en dos o tres tiem. pos. En el primero se efectúa casi siempre la vulrectomía haciendo a los 10 o 12 días las dos linfadenectomías in. ginales simultáneamente, o si esto no, es posible, la de un lado 510 días dispués la del otro en un tercer tiempro. Cuando el cáncer corresponde en la clasificación de Taussig a un estado I o II, éste prefiere hacer primero las linfadenectōmias y luégo la vulvecio. mía.

\section{Vulvectomía.}

Respecto a la extensión de la zona resecada naturalmente que rariará; to acuerdo con el tamaño del tumor cenceroso, pero en todo caso debe exitirparse una área de tejido sano cirenndante de alrededor de una pulgada de ancho $y$ si es muy extenso se extirpa primero con el electrocauterio resecando posteriormente con el bisturí uua zona de piel quemada de media pulçada de ancho. En caso de haber leucoplasia simultáneamente con el carcinoma, deberán extirparse sus placas total. mente para evitar las reincidencias. La zona extirpada incluirá en todo 
and fos grandes y pequeños labios y e! ritoris en su totalidad. Hezando hasta dintroito raginal. Deben respetarse lo his posible el meato uretral y la uretwa misma, para evitar la posterior incurinencia de orina, haciéndose si es massario posteriormente un tratamionta por medio de Radium intrapuretral. 'flie actúra aquí muy bien.

Hecha la extirpación de la lesión. fitoda el problema del afrontamiento th los bordes. ya que por lo extenso iti da resección generalmente se debell Hajar suthras a gran tensión que posfuriormente se esfacelan y necrosan. psprecialmente en la región perineal. tenipndo que hacerse el cierre de la heridi jor seginda intención, empleando injertos de piel. Para obviar esto en parte, Tunksig (61, 62, 63). ideó un procedimiento que él llama de "colgrajo vagiinil". con el cual ha obtenido magnifieos resultados, especialmente en aquellas mujeres de edad no muy avanzada en las" cemales debe tratar de dejarse un introito raginal lo más normal poxible. Comsiste esencialmente en disecar la pared posterior de la vagina. separándola del recto en una distaneia de mos rinue centímetros y luégo hacer un corte lateral a cada lado de la zona desprendida; se obtiene de esta manera un *olgajo que puede ser aproximado sin mucha tensión al borde anal de la he. ricla, al (nal es mido por (natro o eince) pmotos separados de seda o caterut cromado. Tuégo se une el colgajo al piso perineal para eliminar los esparion muertos y a los bordes de la herida, que queda de esta manera cerrada en sil parte posterior sin tensión exagerada. En la parte anterior pneden unirse los bordes de la herida a la zona de mucosa dejada alrededor del meato uretral y el orificio vaginal; pero si ex meuy grande el espacio cruento, putede harerse a cada lado una incisión en angulo, formando así dos colgajos de piel que son desprendidos y unidos 1110 a otro en la línea media, por delante del meato. obturándose así dicha zond. eruenta (ver fig. 4). Pueden emplearse puntos separados de seda y catgut. En nno de nuestros más recientes casos (no incluílo en las estadísticas que presentamos). en que por lo extenso de la lesión nos vimos obligados a exteuder la escisión quirúrgica hasta el rebordo anterior del ano, reconstruimos el periné, en la misma forma que se hace cuando se tiene una laceración de tercer grado, afrontando esfinter anal. elevadores. ete.; y obturimos un afrontamiento de los colgajos cutáneos sorprendentemente fácil. Taussig. no aconseja dejar drenaje. sino en el casi de haberse abierto el espacio subpúbico.

(Contimuarii) 